A3

doi: 10.14232/ syrpharmacognosy.2021.a3

\title{
Cannabinoids: an interesting and promising, but challenging field
}

Ákos Bajtel

Email: bajtel.akos@pharmacognosy.hu

The issue of Cannabis sativa has affected numerous fields of research and has provided space for valuable research possibilities. Its importance as a remedy is unquestionably getting popular nowadays. Due to the unclear legislation, consumers have access to cannabis-containing food supplements in shops and through online websites. Uncontrolled authorisation procedures contribute to the potential health risk factors of these products.

The main goal of our work was to investigate cannabis oil food supplements available on the online market. We developed several separation methods to accomplish full resolution of 14 cannabinoid markers in fifteen commercially available Cannabis products. We carried out a meta-analysis on the safety evaluation of two cannabinoid, nabilone and dronabinol, which are the active substances of prescription drugs approved in several countries. We managed to expand our research to pharmacological and pyrolysis studies of cannabidiol (CBD) in cooperation. We examined the in vitro and in vivo cardiovascular effects of cannabidiol and analysed the pyrolysis products of CBD using standard heating conditions applied in electronic cigarettes.

Supervisors: Dezső Csupor, Tivadar Kiss

\section{Acknowledgements:}

To Prof. András Varró and his research group for the co-operation at the Department of Pharmacology and Pharmacotherapy at the University of Szeged. To Dr Zsuzsanna Czégény and her research group for the co-operation at the Institute of Materials and Environmental Chemistry, Eötvös Lóránd Research Network.

\section{References}

[1] P. Orvos et al., Sci. Rep. 2020; 10(1): 1-9. 